\title{
On Tomography with Limited Data
}

\author{
Fawaz Hiouj \\ Department of Mathematics, The Petroleum Institute, United Arab Emirates \\ E-mail: fhioui@pi.ac.ae
}

Received: September 9, $2016 /$ Accepted: November 16, 2016

\begin{abstract}
We give an algorithm for reconstructing a density function $f$ in the plane from limited number of Radon projections on a range of angles $-\varphi^{*}<\varphi<\varphi^{*}$ together with a few well selected angles outside this range. In doing so, we assume that $f$ is subjected to a linear transformation that produces $\mathrm{g}$, and actually recover $\mathrm{g}$. Interpolation is a basic tool in our calculations
\end{abstract}




\section{Introduction}

In [9], we addressed the important problem of Tomography with Unknown Orientation. In this paper, we consider the problem of two-dimensional parallel beam Tomography with limited data. Limited data problem in tomography arises in different forms and for different reasons. It can be caused by missing data over a certain angle interval, for example in C-arm CT or dental $\mathrm{CT}$, which make an inverse Radon transform a nontraditional tomography.

Literature has plenty different approaches of solving the problem, for example [1]-[7]. An important approach is the theory of using different orthogonal moments, as in $[4,5,6,7]$. Of these for example, [6] extended Wang's method [5] using the orthogonal Legendre moments to improve the quality of the reconstructed image.

Another approach of Reconstruction of image from limited range projections using squashing was presented in [1]. Indeed, our work is related to this particular approach.

We develop an algorithm for reconstructing the density function $f$ in the plane from limited number of Radon projections on a range of angles $-\varphi^{*}<\varphi<\varphi^{*}$ together with a few well selected angles outside this range. In doing so, we assume that $f$ is subjected to a linear transformation that produces $g$. We use the given data [ The Radon Transform of $f$ on the interval $-\varphi^{*}<\varphi<\varphi^{*}$ ]and we employ the relation between $f$ and $g$ (in Radon Transform domain) to deduce the Radon transform of $g$ on a much wider interval $-\theta^{*}<\theta<\theta^{*}$. We also will assume the availability of some more selected projections of $f$ to secure the projections of $g$ outside the range $-\theta^{*}<\theta<\theta^{*}$. In going from $f$ to $g$ we need the basic tools of interpolation. We then can use traditional algorithms to recover $g$ and so is $f$.

In the remaining part of this introduction we introduce notation and a basic formula. Following [11], let $f$ be a 2-D nonnegative function on the $x y$ - plane with a compact support and $\xi=$ $\left(\begin{array}{c}\cos \varphi \\ \sin \varphi\end{array}\right)$ is a unit vector; the Radon Transform of $f$ along the line $L=\{(x, y): x \cos \varphi+$ $y \sin \varphi=p\}$ is given by:

$f^{\vee}(p, \varphi)=f_{\varphi}^{\vee}(p)=\int_{-\infty}^{\infty} f(p \cos \varphi-t \sin \varphi, p \sin \varphi+t \cos \varphi) d t$

We may use the vector form $f^{\vee}(p, \varphi)=f^{\vee}(p, \xi)$ 


\section{Problem Formulation}

Let $f$ be an unknown nonnegative density function with compact support on the $x y$ - plane. We address the problem of recovering $f$ using a 'limited' number of its Radon Projections. However, the term limited here takes different forms due to different applications or mathematical assumptions associated with it. In traditional Tomography, of course, we have a full range of projections $f^{\vee}(p, \varphi)$ over 0 to 180 with fine uniformly spaced values of the argument $p$ given at $p_{1}, p_{2}, \ldots p_{m}$. Examples of variations of mathematical assumptions include: only few projections are available, or projections are available on a limited range of angles, for example as in [1]: 0 to 160 instead of 0 to 180 ; and so on.

In this paper, we consider the problem of construction assuming that projections are known on a range of angles $-\varphi^{*}<\varphi<\varphi^{*}$ for some acute angel $\varphi^{*}$

\section{Proposed Approach}

We organize this section in three parts:

A. We show an approach of employing interpolation to recover unknown projections from a known set of projections.

B. We review the theory of Radon Transform of linear transformation.

C. We describe the mathematical bases of our reconstruction approach.

\section{A. Local Interpolation}

Let $f$ be nonnegative function on the $x y$-plane that vanishes outside the unit disk. Assume that we have $N$ projections $f^{\vee}\left(p, \varphi_{j}\right), j=1, \ldots N$ so that $\left\{\varphi_{j}\right\}$ is a fine sample- $\varphi^{*}<\varphi_{j}<\varphi^{*}$, with fine uniformly spaced values of the argument $p$ given at $p_{1}<p_{2}<\cdots<p_{m}$. For example, say the range $-50^{\circ}$ to $50^{\circ}$. Our goal is to approximate $f^{\vee}(p, \varphi)$ for any angle $\varphi$ and value $p$ with $-\varphi^{*}<\varphi<\varphi^{*} ;-1<p<1$. We can accomplish this using two dimensional interpolation. We build this approach on the following fact that was proved in [8]. 


\section{Theorem 1}

Suppose $f \in L_{2}\left(R^{2}\right)$ and that $f$ vanishes outside the unit disk.

Then, $f_{\varphi}^{\vee}$ is in $L_{2}(R)$ for all $\varphi$ and $\left\|f_{\varphi_{1}}^{\vee}-f_{\varphi_{2}}^{\vee}\right\|$ tends to zero as $\varphi_{1}$ approaches $\varphi_{2}$. In other words, the map $\varphi \rightarrow f_{\varphi}^{\vee}$ is a continuous map from $S^{1}$ to $L_{2}(R)$, where $S^{1}$ is the unit circle.

We consider the sonogram on a rectangular grid, as in Figure $1 a$, on which the $x$ axis represent the angles $-\varphi^{*}<\varphi_{j}<\varphi^{*}$, and the $y$ - axis represent the values $\boldsymbol{p}_{1}, \boldsymbol{p}_{2}, \ldots \boldsymbol{p}_{\boldsymbol{m}}$. Let

$\mathcal{A} \boldsymbol{f}^{\vee}(\boldsymbol{p}, \boldsymbol{\varphi}) \approx \boldsymbol{f}^{\vee}(\boldsymbol{p}, \boldsymbol{\varphi})$

be the interpolating function. Nearest neighbor interpolation, bilinear interpolation, spline interpolation or others can be used. However, in computing $\mathcal{A} f^{\vee}(\boldsymbol{p}, \boldsymbol{\varphi})$ we interpolate $\boldsymbol{f}^{\vee}(\boldsymbol{p}, \boldsymbol{\varphi})$ only on the vertical strip of the sinogram defined by $\varphi_{j}$ and $\varphi_{j+1}$ such that $\varphi_{j} \leq \varphi \leq \boldsymbol{\varphi}_{j+1}$. Our experiment section shows more details on implementation and reliability.

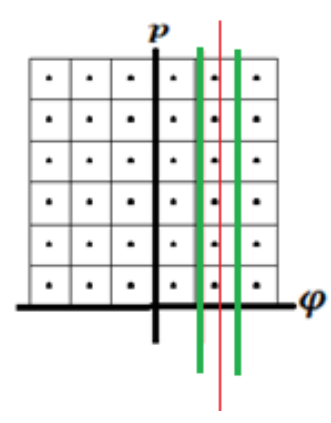

(1a)

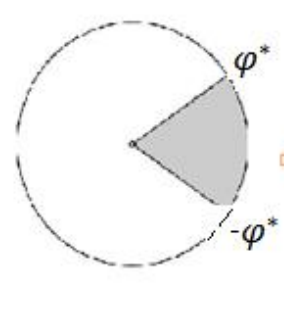

(1b)

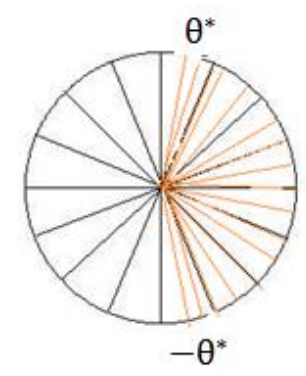

Figure 1. a: a rectangular grid on which the horizontal axis represents the angles $-\varphi^{*}<\varphi_{\mathrm{j}}<$ $\varphi^{*}$, and the vertical axis represents the values $p_{1}, p_{2}, \ldots p_{m}$. b: Transformation (6) maps range of angles $-\theta^{*} \leq \theta \leq \theta^{*}$ to a prescribed (available) range of angles $-\varphi^{*}<\varphi<\varphi^{*}$

\section{B. Using Linear Transformations}

Let $f$ be nonnegative function on the $x y$ - plane with a compact support. Let $A$ be a $2 \times 2$ nonsingular matrix and let

$g(x, y):=f\left[A(x, y)^{T}\right]$,

Then, as in our study in [10], we have 
$g^{\vee}(p, \xi)=\frac{\Delta}{|\mu|} f^{\vee}\left(\frac{p}{|\mu|}, \frac{\mu}{|\mu|}\right)$

Where $\mu=B^{T} \xi ; \quad \mathrm{B}=A^{-1}, \Delta=|\operatorname{det}(B)|$, and $|\mu|$ is the magnitude of the vector $\mu$.To write (4) in the standard scalar form, we define angles $\theta$ and $\varphi$ such that $\xi=\left(\begin{array}{l}\cos \theta \\ \sin \theta\end{array}\right)$ and $\mu=B^{T} \xi=|\mu|\left(\begin{array}{c}\cos \varphi \\ \sin \varphi\end{array}\right)$

So we have,

$g^{\vee}(p, \theta)=\frac{\Delta}{|\mu|} f^{\vee}\left(\frac{p}{|\mu|}, \varphi\right)$

Clearly, both $\varphi$ and $\mu$ are functions of $\theta$.

We choose a transformation $A=\left[\begin{array}{ll}1 & 0 \\ 0 & \delta\end{array}\right]$ so that (6) maps the range of angles $-\theta^{*} \leq \theta \leq \theta^{*}$ to a prescribed range of angles $-\varphi^{*}<\varphi<\varphi^{*}$, that is visually explained in Figure $1 \mathrm{~b}$. As an example: when $\boldsymbol{\delta}=5$

then, this transformation (approximately) maps the range $-50^{\circ}<\varphi<50^{\circ}$ to the range $-80^{\circ}<\theta<80^{\circ}$ as calculated in Table 3 of the next section.

\section{C. Proposed Algorithm}

To recover $f$ from a given set of projections, we actually want to recover $g$ as in (3); with a choice of $\delta$. Visibility and resolution of our reconstruction issues will be discussed shortly. We apply a standard reconstruction algorithm such as the Filtered Back projection method on $g^{\vee}(p, \theta)$ estimated on a full range of $-89^{\circ}<\theta \leq 90^{\circ}$ incremented by $1^{\circ}$; as well as a fine sample of the argument $-1<p<1$.In doing so, we require two types of input data:

A. $\quad$ projections of $f$ are known on a range of angles $-\varphi^{*}<\varphi<\varphi^{*}$

B. projections of $f$ are known for those angles $\varphi_{k}$ that are corresponding to angles $\theta_{k}$ through (5) where $\theta_{k}$ is an angel outside the range of $-\theta^{*} \leq \theta \leq \theta^{*}$ We then consider the following algorithm of two steps:

\section{Algorithm 1:}

Step 1: As explained previously, assume that the ranges of angles $-\theta^{*} \leq \theta \leq \theta^{*}$ and $-\varphi^{*} \leq$ $\varphi \leq \varphi^{*}$, are related through (6), then $g^{\vee}(p, \theta)$ on $-\theta^{*} \leq \theta \leq \theta^{*}$ can be calculated by (6) [by actually interpolating $f^{\vee}(p, \varphi)$ on range of angles $-\varphi^{*}<\varphi<\varphi^{*}$. 
Step 2: For angles $\theta_{k}$ outside the range of angles $-\theta^{*} \leq \theta \leq \theta^{*}$ we use (6) to obtain $g^{\vee}(p, \theta)$ from $f^{\vee}(p, \varphi)$.

In the next section, we analyze these steps and its limitation with a closer look at the different parameters involved, such as the choice of $\delta$ or sampling the arguments $p$.

\section{Discussion and Examples}

We now test these ideas and algorithms looking at the different parameters involved, such as the choice of $\delta$, or sampling the argument $p$ and others. First, we tested our interpolation approach on several types of images and we show two of these:

in figure $2 \mathrm{a}$ we consider the block image $\mathrm{E}$ for which we compute $\mathcal{A} f^{\vee}(p, \varphi)$ and $f^{\vee}(p, \varphi)$ as in (2)for some random angles $\varphi$. Reslts are shown in table 1 , and displayed visually in figures $2 \mathrm{~b}-2 \mathrm{f}$

Table 1. Comparing $\mathcal{A} f^{\vee}(p, \varphi)$ and $f^{\vee}(p, \varphi)$ for the E image.

\begin{tabular}{llll}
\hline Angle $\varphi^{\circ}$ & $\left\|\mathcal{A f}^{\vee}(\boldsymbol{p}, \varphi)-\boldsymbol{f}^{\vee}(\boldsymbol{p}, \boldsymbol{\varphi})\right\|$ & $\left\|\boldsymbol{f}^{\vee}(\boldsymbol{p}, \boldsymbol{\varphi})\right\|$ & Figure 2 \\
\hline-14.00 & 0 & 28.0609 & $\mathrm{~B}$ \\
-10.70 & 0.0792 & 28.8047 & $\mathrm{C}$ \\
0 & 0 & 31.5535 & $\mathrm{D}$ \\
3.500 & 0.2846 & 30.6898 & $\mathrm{E}$ \\
13.500 & 0.0814 & 28.1601 & $\mathrm{~F}$ \\
\hline
\end{tabular}




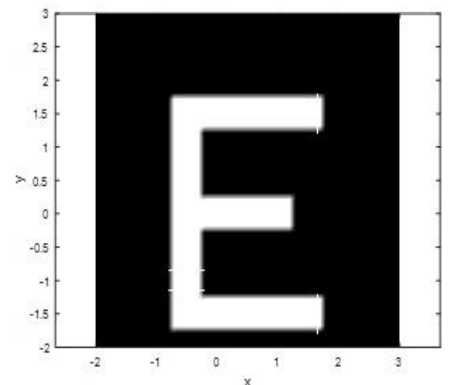

Fig2a

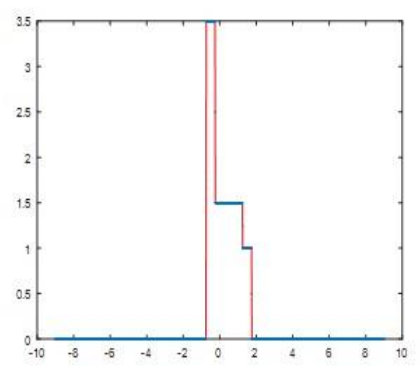

Fig2d

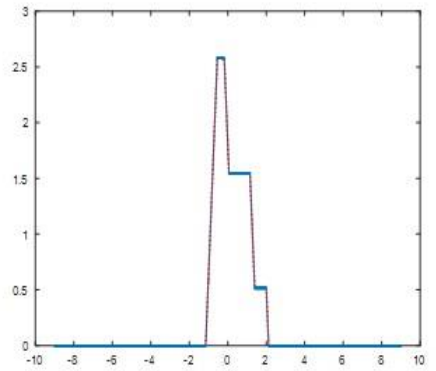

Fig $2 b$

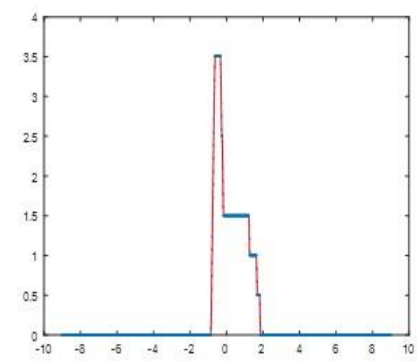

Fig2e

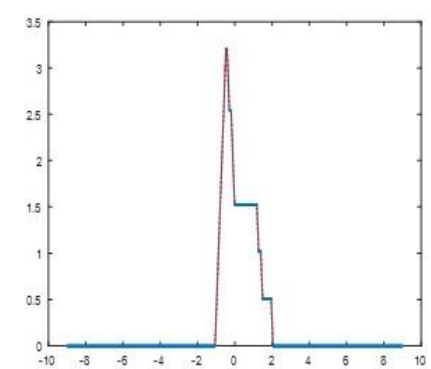

Fig2C

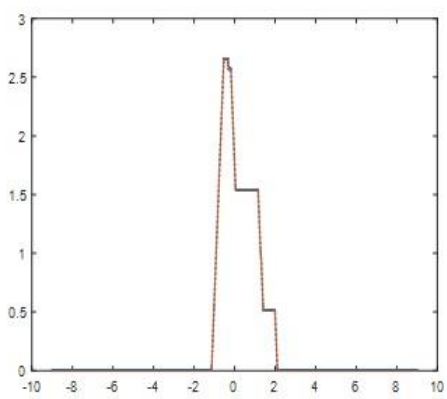

Fig2f

Figure 2. a: original block image E. b-f: selected profiles $f^{\vee}(p, \varphi)$ together with their $\mathcal{A} f^{\vee}(p, \varphi)$

We performed similar calculations on the Shepp-Logan image and show the results in table 2 and figure 3.

Table 2. Comparing $\mathcal{A} f^{\vee}(p, \varphi)$ and $f^{\vee}(p, \varphi)$ for the $\mathrm{E}$ image.

\begin{tabular}{llll}
\hline Angle $\varphi^{\circ}$ & $\left\|\mathcal{A f}^{\vee}(\boldsymbol{p}, \boldsymbol{\varphi})-\boldsymbol{f}^{\vee}(\boldsymbol{p}, \boldsymbol{\varphi})\right\|$ & $\left\|\boldsymbol{f}^{\vee}(\boldsymbol{p}, \boldsymbol{\varphi})\right\|$ & Figure 3 \\
\hline-6.7 & 4.7690 & 0.0217 & $\mathrm{~B}$ \\
0 & 4.7748 & 0 & $\mathrm{C}$ \\
3.3 & 4.7681 & 0.0210 & $\mathrm{D}$ \\
11.9 & 4.7225 & 0.0105 & $\mathrm{E}$ \\
\hline
\end{tabular}




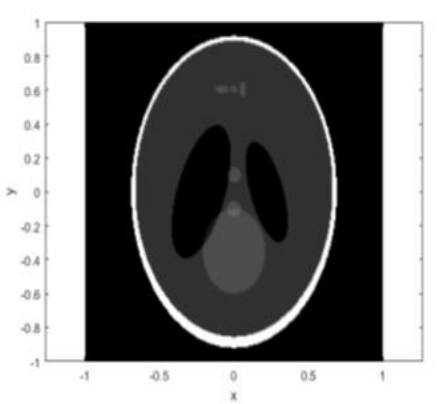

Fig $3 a$

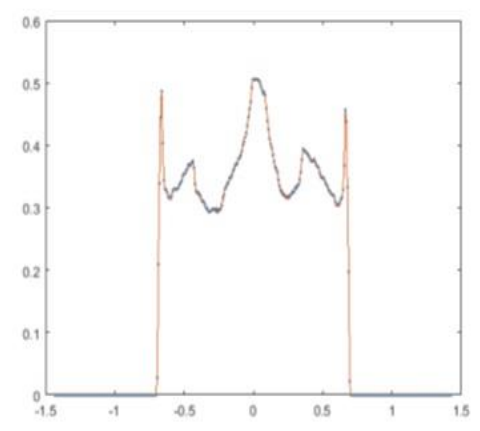

Fig 3d

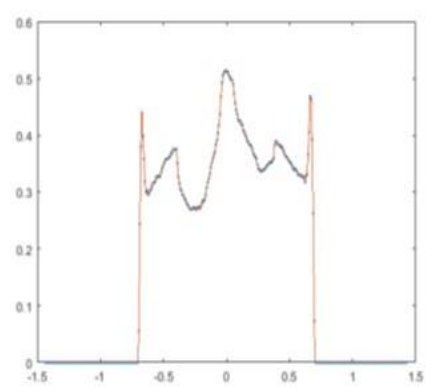

Fig $3 b$

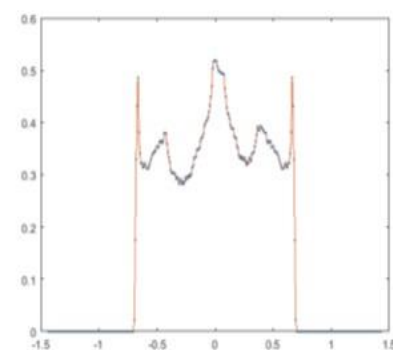

Fig 3c

Figure 3. a: original phantom-Shepp-Logan. b-e: selected profiles $f^{\vee}(p, \varphi)$ together with their $\mathcal{A} f^{\vee}(p, \varphi)$.

We now apply our algorithm on the block image $F$ Shown in figure 4 a. Then, for the transformation $A=\left[\begin{array}{ll}1 & 0 \\ 0 & 5\end{array}\right]$ as shown in figure $4 \mathrm{~b}$, we require that $g^{\vee}\left(p, \theta_{k}\right)$ is accessible on the range $-80^{\circ} \leq \theta \leq 80^{\circ}$ and increment of $1^{\circ}$. Indeed, we show in table 3 all values of $\theta_{k}$ in the full range of $-90^{\circ} \leq \theta_{k} \leq 89^{\circ}$ and the corresponding values of $\varphi_{k}$ as in equation (6), when $\delta=5$. We see from this table that for the range $-80^{\circ} \leq \theta \leq 80^{\circ}$ we need $\varphi^{*} \cong 50^{\circ}$. For angles $\theta_{k}$ outside the range of angles $-80 \leq \theta \leq 80$ we use (6) to obtain $g^{\vee}\left(p, \theta_{k}\right)$ from $f^{\vee}\left(p, \varphi_{k}\right)$ where these $\varphi_{k}$ angles are shown in table 3. In Figure 4c, we show the recovered $g$ using our algorithm. If we repeat the above calculations with the transformation $A=\left[\begin{array}{cc}1 & 0 \\ 0 & 10\end{array}\right]$ as figure $4 \mathrm{~d}$, then, for $g^{\vee}\left(p, \theta_{k}\right)$ to be accessible on the range $-80^{\circ} \leq \theta \leq 80^{\circ}$ we need $\varphi^{*} \cong 30^{\circ}$. Output of algorithm (1) is shown in figure 4e. A similar calculation is performed on the Shepp-Logan image with results in figure 5 . 


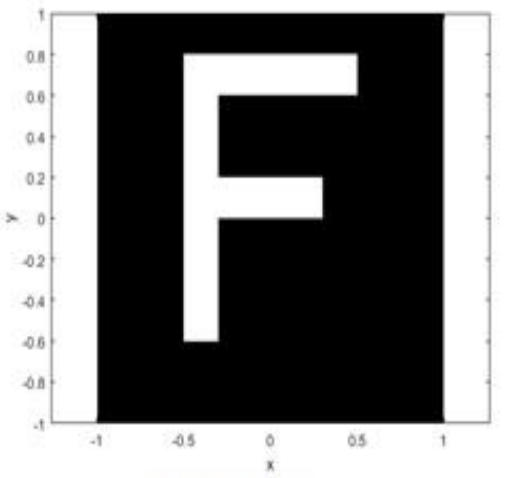

Fig $4 \mathrm{a}$

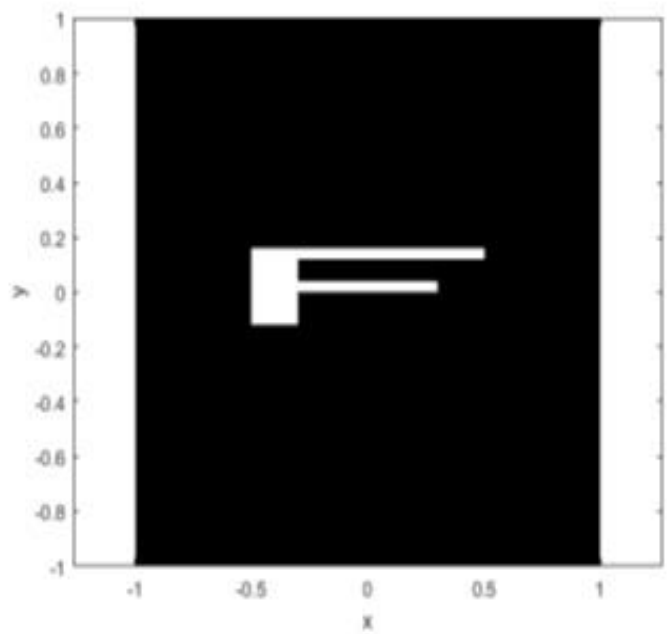

Fig $4 b$

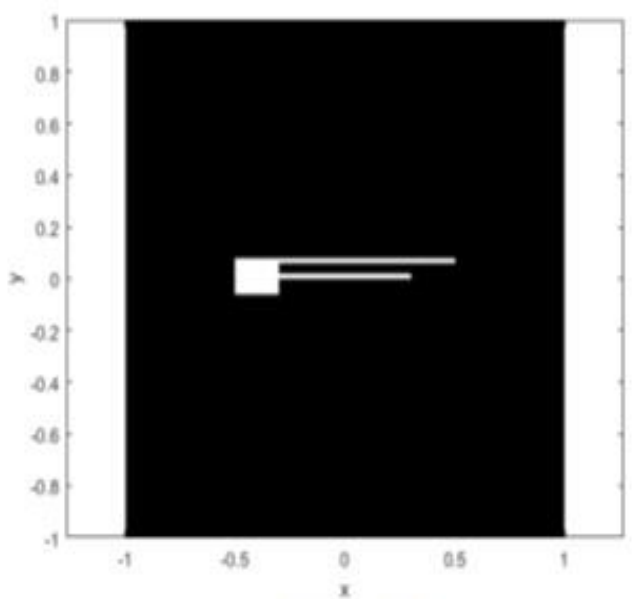

Fig $4 \mathrm{~d}$

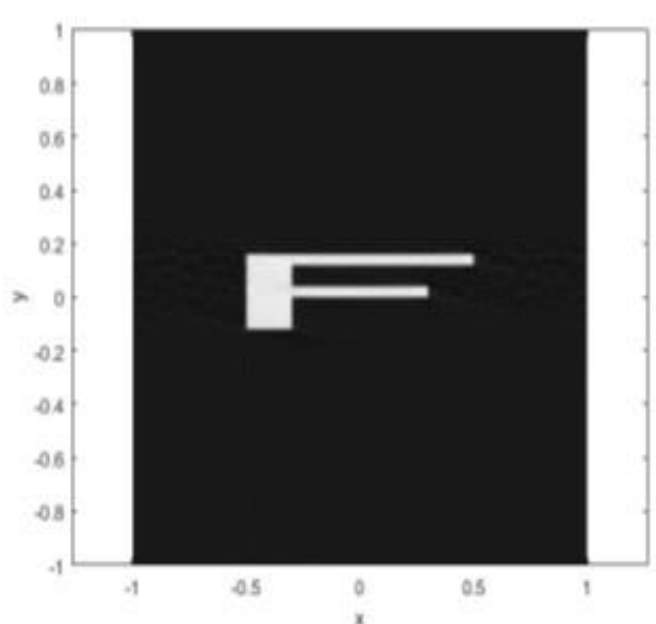

Fig $4 \mathrm{C}$

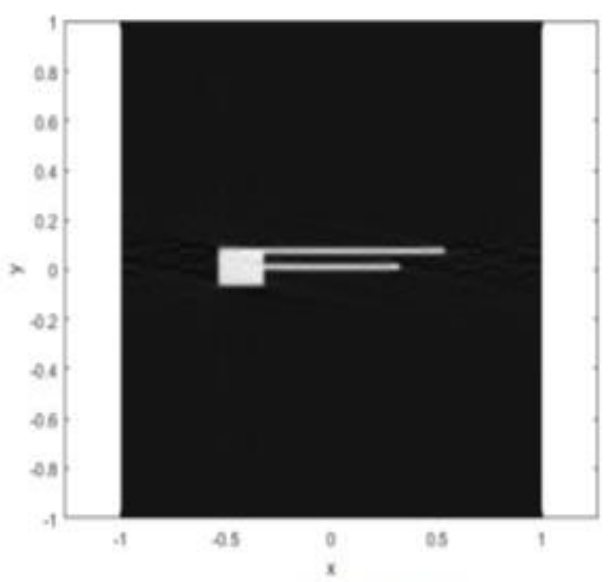

Fig $4 \mathrm{e}$

Figure 4. a. original Block b. Transformation $g(x, y)$ with $\delta=5$. c.recover $g(x, y)$. d. Transformation $\mathrm{g}(\mathrm{x}, \mathrm{y})$ with $\delta=10$. e. $\operatorname{Recover} \mathbf{g}(\mathbf{x}, \mathbf{y})$. 


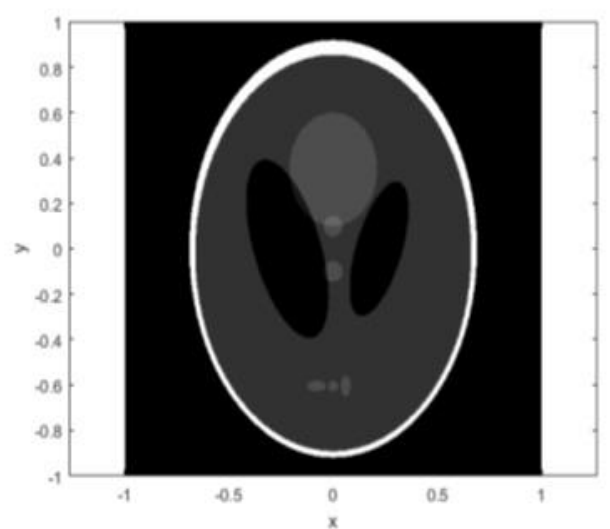

Fig $5 a$

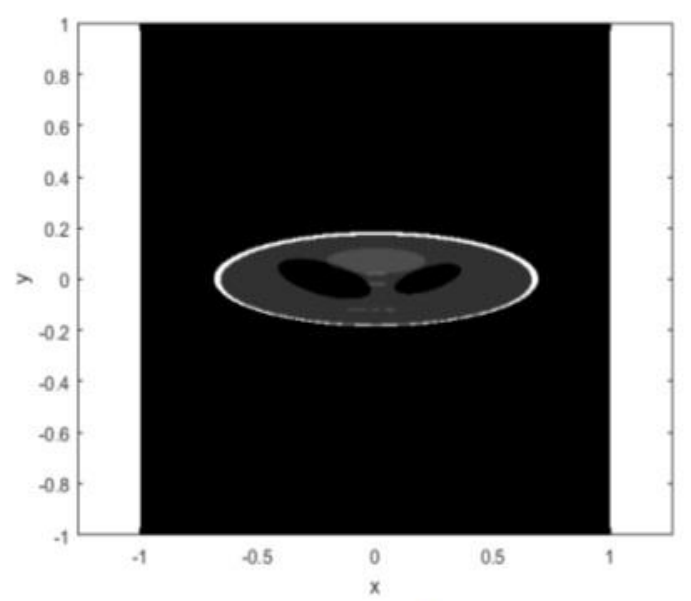

Fig 5b

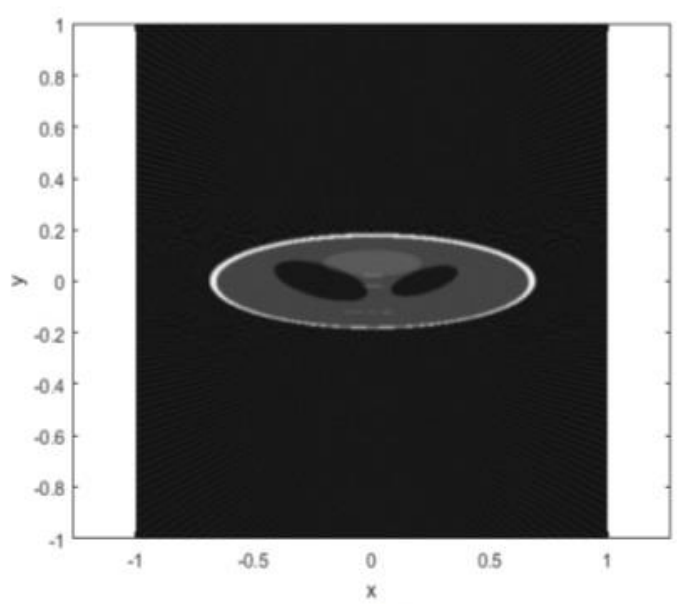

Fig $5 \mathrm{C}$

Figure 5. a. original image b. Transformation $g(x, y)$ with $\delta=5$. c. Recover $g(x, y)$

In the above experiments, we assumed that images are defined on the unit circle with 250 values of the spacing variable $p$.

A finer sampling of $p$ would produce better results.

Also, we see that with $\delta=5$ we need to have the projections of $f$ on the range $-50^{\circ}<\varphi<50^{\circ}$ which is 101 projections, plus additional 19 projection of $f$ that correspond to angles $\theta$ outside the range $-80^{\circ} \leq \theta \leq 80^{\circ}$. That is a total of 120 projections.

Less number of projections is still possible, for if $\delta=10$, we would need the projections of $f$ on the range $-30^{\circ}<\varphi<30^{\circ}$

Plus the outside range of 19 , which is 80 projections in total.

But when $\delta$ becomes large, we would face resolution and practical issues of reconstruction. 
Table 3. Values of $-90^{\circ} \leq \theta_{k} \leq 89^{\circ}$ and the corresponding values of $\varphi_{k}$ as in equation (6) when $\delta=5$.

\begin{tabular}{|c|c|c|c|c|c|c|c|}
\hline$\theta \mathbf{k}$ & $\phi \mathbf{k}$ & $\theta \mathbf{k}$ & $\phi k$ & $\theta \mathbf{k}$ & $\phi \mathbf{k}$ & $\theta \mathbf{k}$ & $\phi \mathbf{k}$ \\
\hline-89 & -85.0121 & -44 & -10.9314 & 1 & 0.2 & 46 & 11.7009 \\
\hline-88 & -80.0958 & -43 & -10.5645 & 2 & 0.4002 & 47 & 12.1051 \\
\hline-87 & -75.3164 & -42 & -10.2085 & 3 & 0.6005 & 48 & 12.5234 \\
\hline-86 & -70.7286 & -41 & -9.8627 & 4 & 0.8013 & 49 & 12.9568 \\
\hline-85 & -66.3733 & -40 & -9.5266 & 5 & 1.0024 & 50 & 13.4063 \\
\hline-84 & -62.2771 & -39 & -9.1996 & 6 & 1.2042 & 51 & 13.8732 \\
\hline-83 & -58.4533 & -38 & -8.8811 & 7 & 1.4067 & 52 & 14.3587 \\
\hline-82 & -54.9041 & -37 & -8.5706 & 8 & 1.6101 & 53 & 14.8641 \\
\hline-81 & -51.6235 & -36 & -8.2677 & 9 & 1.8143 & 54 & 15.391 \\
\hline-80 & -48.5995 & -35 & -7.9719 & 10 & 2.0197 & 55 & 15.9409 \\
\hline-79 & -45.8164 & -34 & -7.6829 & 11 & 2.2263 & 56 & 16.5157 \\
\hline-78 & -43.2567 & -33 & -7.4002 & 12 & 2.4343 & 57 & 17.1174 \\
\hline-77 & -40.9022 & -32 & -7.1235 & 13 & 2.6437 & 58 & 17.7481 \\
\hline-76 & -38.7351 & -31 & -6.8525 & 14 & 2.8547 & 59 & 18.4103 \\
\hline-75 & -36.738 & -30 & -6.5868 & 15 & 3.0675 & 60 & 19.1066 \\
\hline-74 & -34.8951 & -29 & -6.3261 & 16 & 3.2823 & 61 & 19.8399 \\
\hline-73 & -33.1915 & -28 & -6.0701 & 17 & 3.4991 & 62 & 20.6136 \\
\hline-72 & -31.6138 & -27 & -5.8186 & 18 & 3.7181 & 63 & 21.4311 \\
\hline-71 & -30.1498 & -26 & -5.5714 & 19 & 3.9395 & 64 & 22.2966 \\
\hline-70 & -28.7886 & -25 & -5.3281 & 20 & 4.1634 & 65 & 23.2146 \\
\hline-69 & -27.5203 & -24 & -5.0885 & 21 & 4.3901 & 66 & 24.19 \\
\hline-68 & -26.3362 & -23 & -4.8525 & 22 & 4.6198 & 67 & 25.2284 \\
\hline-67 & -25.2284 & -22 & -4.6198 & 23 & 4.8525 & 68 & 26.3362 \\
\hline-66 & -24.19 & -21 & -4.3901 & 24 & 5.0885 & 69 & 27.5203 \\
\hline-65 & -23.2146 & -20 & -4.1634 & 25 & 5.3281 & 70 & 28.7886 \\
\hline-64 & -22.2966 & -19 & -3.9395 & 26 & 5.5714 & ו7 & 30.1498 \\
\hline-63 & -21.4311 & -18 & -3.7181 & 27 & 5.8186 & 72 & 31.6138 \\
\hline-62 & -20.6136 & -17 & -3.4991 & 28 & 6.0701 & 73 & 33.1915 \\
\hline-61 & -19.8399 & -16 & -3.2823 & 29 & 6.3261 & 74 & 34.8951 \\
\hline-60 & -19.1066 & -15 & -3.0675 & 30 & 6.5868 & 75 & 36.738 \\
\hline-59 & -18.4103 & -14 & -2.8547 & 31 & 6.8525 & 76 & 38.7351 \\
\hline-58 & -17.7481 & -13 & -2.6437 & 32 & 7.1235 & 77 & 40.9022 \\
\hline-57 & -17.1174 & -12 & -2.4343 & 33 & 7.4002 & 78 & 43.2567 \\
\hline-56 & -16.5157 & -11 & -2.2263 & 34 & 7.6829 & 79 & 45.8164 \\
\hline-55 & -15.9409 & -10 & -2.0197 & 35 & 7.9719 & 80 & 48.5995 \\
\hline-54 & -15.391 & -9 & -1.8143 & 36 & 8.267 & 781.000 & $0 \quad 51.6235$ \\
\hline-53 & -14.8641 & -8 & -1.6101 & 37 & 8.57 & 682.00 & $00 \quad 54.9041$ \\
\hline-52 & -14.3587 & -7 & -1.4067 & 38 & 8.881 & 183.00 & $00 \quad 58.4533$ \\
\hline
\end{tabular}




\begin{tabular}{llllllllll}
-51 & -13.8732 & -6 & -1.2042 & 39 & 9.199 & 6 & 84.00 & 00 & 62.2771 \\
-50 & -13.4063 & -5 & -1.0024 & 40 & 9.526 & 6 & 85.00 & 00 & 66.3733 \\
-49 & -12.9568 & -4 & -0.8013 & 41 & 9.862 & 7 & 86.00 & 00 & 70.7286 \\
-48 & -12.5234 & -3 & -0.6005 & 42 & 10.208 & 5 & 87.000 & 0 & 75.3164 \\
-47 & -12.1051 & -2 & -0.4002 & 43 & 10.564 & 5 & 88.000 & 0 & 80.0958 \\
-46 & -11.7009 & -1 & -0.2 & 44 & 10.931 & 4 & 89.000 & 0 & 85.0121 \\
-45 & -11.3099 & 0 & 0 & 45 & 11.3099 & 90.0000 & 90.0000 \\
\hline
\end{tabular}

\section{Conclusion}

In this article, we addressed the problem of recovering an image from limited number of its Radon Transform. Indeed, we proposed an algorithm for reconstructing the density function $f$ with Radon projections on a range of angles $-\varphi^{*}<\varphi<\varphi^{*}$ together with a few well selected angles outside this range. In doing so, we assume that $f$ is subjected to a linear transformation that produces $g$. We use the given data [The Radon Transform of $f$ on the interval $-\varphi^{*}<\varphi<\varphi^{*}$ ]and we employ the relation between $f$ and $g$ (in Radon Transform domain) to deduce the Radon transform of $g$ on a much wider interval $-\theta^{*}<\theta<\theta^{*}$. We also assumed that projections of $f$ that are corresponding to the projections of $g$ outside the range $-\theta^{*}<\theta<\theta^{*}$ are given. In computing the projections of $\mathrm{g}$, we used basic tools of interpolation to approximate the projections of $f$. We then used traditional algorithms to recover $g$.

\section{References}

[1] Reeds JA and LA Shepp (1987) Limited angle reconstruction in tomography via squashing. IEEE Trans. on Med. Imaging 6: 89-97.

[2] Prince JL and AS Willsky (1993). Hierarchical reconstruction using geometry and sinogram restoration. IEEE Trans. on Image Proc 2 (3): 40-416.

[3] Luo J, W Li, and Y Zhu (2010). Reconstruction from limited-angle projections based on spectrum analysis. IEEE Transactions on Image Processing 19 (1): 131-140.

[4] Teague MR (1980) Image analyis via the general theory of moments. J. Opt. Soc. Amer. 70 (8): 920-930.

[5] Wang TJ and TW Sze (2001) The image moment method for the limited range CT imagereconstruction and pattern recognition. Pattern Recognit. 34 (1 1): 2145-2154. 
[6] Shu HZ, J Zhou, GN Han, LM Luo, and JL Coatrieux (2007) Image reconstruction from limited range projections using orthogonal moments. Pattern Recognit. 40 (2): 670-680. [7] Dai XB, HZ Shu, LM Luo, GN Han, and JL Coatrieux (2009) Reconstruction of tomographic image from limited range projections using discrete Radon transform and Tchebichef moments. IEEE Trans Image Process.

[8] Singer A and H Wu (2013) Two-dimensional tomography from noisy projections taken at unknown random directions. SIAM J Imaging Sc 6 (1): 136-175.

[9] Hiouj F (2015) On Tomography with Unknown Orientation. Journal of Mathematical Sciences \& Computer Applications 2 (2): 125-136, 2015 doi: 10.5147/imsca.2015.0178. [10] Hjouj F (2011) Linear Transformation Recognition Using Radon Transform. Journal of Mathematical Sciences \& Computer Applications 201 1, 1 (2): 40-47. doi: 10.5147 /imsca.2011.0038.

[1 1] Deans SR (1983). The Radon Transform and Some of Its Applications. New York, John Wiley\& Sons, Inc. 